\title{
Determination of Solubility of 4-(2-Hydroxyethyl)-1-piperazi neethanesulfonic Acid and its Sodium Salt in Acetonitrile and Voltammetric Investigation of Sulphonamide Drugs in Different Solvents in Their Absence and Presence
}

\author{
László Kiss ${ }^{1,2} \cdot$ Hiba Mohamed Ameen ${ }^{3} \cdot$ Beáta Lemli ${ }^{3}$ Sándor Kunsági-Máté2,3
}

Received: 9 March 2020 / Accepted: 6 October 2020 / Published online: 20 January 2021

(C) The Author(s) 2021

\begin{abstract}
Sulphonamide drugs (sulphamethazine, sulphamerazine, sulphadiazine, sulphathiazole) were studied in a wide potential window (between 2 and $-2 \mathrm{~V}$ ) in acetonitrile, dimethyl sulphoxide and in 50-50 v/v\% binary mixtures of acetonitrile and water. The voltammograms of the outlined compounds were very similar both in the anodic and cathodic part in each non-aqueous solvents except for sulphathiazole. These sulphonamide drugs were also investigated in presence of 4-(2-hydroxyethyl)-1-piperazineethanesulfonic acid (HEPES) and its sodium salt and the voltammograms changed due to an acid-base reaction. HEPES and its sodium salt could be investigated in acetonitrile only in their saturation concentration as they were slightly soluble in this solvent. In a separate experiment their solubilities were determined at $298 \mathrm{~K}$ in acetonitrile with the co-solvent calibration method using water as co-solvent. Complementary fluorescence studies in dimethyl sulphoxide did not show the presence of any interaction between sulphonamide drugs and HEPES as well as its sodium salt.
\end{abstract}

Keywords 4-(2-Hydroxyethyl)-1-piperazineethanesulfonic acid · Sulphonamide · Acetonitrile $\cdot$ Dimethyl sulphoxide $\cdot$ Fluorescence

\section{Introduction}

The compounds HEPES and its sodium salt are used to make buffers, as they are suitable in living organisms at maintaining physiological $\mathrm{pH}$ also in the presence of carbon dioxide. This buffer was used in a vast number of studies. In many intra-and extracellular

László Kiss

kissl@gamma.ttk.pte.hu

1 Department of General and Physical Chemistry, University of Pécs, Ifjúság 6, Pecs 7624, Hungary

2 János Szentágothai Research Center, Ifjúság útja 20, Pecs 7624, Hungary

3 Department of Organic and Pharmacological Chemistry, University of Pécs, Honvéd street 1, Pecs 7624, Hungary 
electrochemical studies it served as an appropriate choice [1-4] and in protein based detection applications $[5,6]$.

The interaction of HEPES and its conjugate base with the different drugs used in the treatment of a great number of diseases might be useful in other respects. HEPES contains a sulphonic acid group and two tertiary nitrogens in the piperazine moiety, which is the basic part so it is a zwitterionic molecule. The $\mathrm{p} K_{\mathrm{a}}$ of 3 attributed to the first dissociation step makes it capable of acid-base reactions with other molecules having basic functional groups. The second dissociation step has a $\mathrm{p} K_{\mathrm{a}}$ value around 7.5 which is very close to the physiological pHs. Therefore the monosodium salt may also interact with certain molecules. The aim of these studies was the examination of both acidic and basic component of the buffer with potential molecules having basic groups important in medical science.

Sulphonamide drugs are widely used as antibiotics to treat infections and compounds from this family studied here are collected in Fig. 1. Sulphathiazole proved efficient against gonorrhea and sulphadiazine in the treatment of toxoplasmosis. Sulphamethazine and sulphamerazine inhibit the synthesis of dipholic acid necessary for nucleotide synthesis for bacteria thus preventing bacterial growth. Basically sulphonamides have a weak acidic character due to the - NH- group in the neighbourhood of the strongly electron withdrawing $-\mathrm{SO}_{2}-$ group and aromatic $-\mathrm{NH}_{2}$ group if it is protonated so they are able to dissociate in aqueous solutions. Therefore acid-base reactions can occur in presence of other molecules. On the other hand they contain also an $-\mathrm{NH}_{2}$ group in the $p$-aminophenyl moiety which may act as an oxidizable group.

Many sulphonamide drugs are scarcely soluble in water but they can be dissolved in $\mu \mathrm{mol} \cdot \mathrm{L}^{-1}$ concentrations in a wide $\mathrm{pH}$ range and in medical applications these concentrations have attracted interest. Therefore many analytical procedures have been developed for sulphonamide drugs to detect them in blood plasma. In the majority of electrochemical investigations glassy carbon and carbon based electrodes are used with and without modifying layer [7-17] but some works utilize the mercury electrode in electroreduction processes $[18,19]$. Regarding the direct electrochemical reaction of sulphonamides no work is found in the literature where platinum electrode acted as working electrode. Platinum has a low hydrogen evolution overpotential compared with other electrodes so electroreduction of acidic protons present in different molecules can be utilized to get more insight into processes taking place in solutions.

Due to the limited solubility of sulphonamides in water in the $\mathrm{mmol} \cdot \mathrm{L}^{-1}$ concentration range there is a need for investigating these materials rather in nonaqueous solvents and/or in their binary mixtures with water. To obtain significant voltammetric peaks it is advantageous to study these antibiotics in the $\mathrm{mmol} \cdot \mathrm{L}^{-1}$ concentration range particularly if their interaction is in focus with other molecules.

Usually acetonitrile and dimethyl sulfoxide are good solvents for many organic materials they provide an appropriate medium for experiments as the solutes in question can be dissolved in the desired concentration necessary for the experiment. They are miscible with water and have relatively high dielectric constants suitable for voltammetry also in absence of purposely added supporting electrolyte. Acetonitrile has a wide potential window in both anodic and cathodic potential ranges so processes where proton reduction is involved might be studied due to the lack of overlap with the reduction of solvent. The aprotic dimethyl sulphoxide has a low toxicity so these two solvents were selected for investigating the outlined compounds. On the other hand dimethyl sulphoxide is appropriate for the good solvation of cations and cationic moieties of larger molecules. One of the most frequently studied family of drugs is that of sulphonamides. Their interaction with other molecules has increasing medical applications which is the subject of the present work. 
Fig. 1 Structural formula of the investigated HEPES and sulphonamide drugs
HEPES:<smiles>O=S(=O)(O)CCN1CCN(CCO)CC1</smiles>

Sulphadiazine:<smiles>Nc1ccc(S(=O)(=O)Nc2ncccn2)cc1</smiles>

Sulphamerazine:<smiles>Cc1ccnc(NS(=O)(=O)c2ccc(N)cc2)n1</smiles>

Sulphamethazine:<smiles>Cc1cc(C)nc(NS(=O)(=O)c2ccc(N)cc2)n1</smiles>

Sulphathiazole:<smiles>Nc1ccc(S(=O)(=O)/N=c2/[nH]ccs2)cc1</smiles><smiles>Nc1ccc(S(=O)(=O)Nc2nccs2)cc1</smiles> 


\section{Experimental}

To carry out the voltammetric experiments a $1 \mathrm{~mm}$ in diameter platinum disc was used as working electrode, a platinum wire served as counter electrode and a silver wire as reference electrode connected to a potentiostat in a three electrode cell (Dropsens, Spain). The surface of the working electrode was polished with $0.05 \mu \mathrm{m}$ alumina, ultrasonicated in deionized water to remove polishing residue and finally washed with deionized water thoroughly. It was followed by drying before measurements to avoid introduction of water into the non-aqueous solutions. Tetrabutylammonium perchlorate (TBAP) was the supporting electrolyte in all voltammetric studies in non-aqueous systems. The conductivity measurements were carried out with a Mettler Toledo conductometer. The absorbance spectra were taken with a Specord Analytik Jena spectrometer, the fluorescence measurements were carried out with a Fluorolog3 Horiba Jobin-Yvon spectrofuorimeter (Lille, France). For the spectroscopic studies $1 \times 1 \mathrm{~cm}$ quartz cuvettes were used. The temperature for all studies was $25^{\circ} \mathrm{C}$.

The solutions of HEPES and its sodium salt were stored till the measurements in darkness due to their light sensitivity as during their exposure to light produces hydrogen peroxide while they are oxidized [20].

\section{Results and Discussion}

\subsection{Voltammetric Studies of HEPES and its Sodium Salt in Acetonitrile and Dimethyl Sulphoxide}

In order to investigate HEPES and its sodium salt voltammograms were recorded in their solutions prepared with acetonitrile and dimethyl sulfoxide with a platinum electrode. The curves are displayed in Fig. 2 for the two solutes in acetonitrile and in Fig. 3 in dimethyl sulphoxide. They clearly show that the voltammogram of solvent acetonitrile is very similar to the ones recorded in acetonitrile solutions of HEPES and its sodium salt showing that they do not have any characteristic peak neither in the anodic nor in the cathodic potential window. The reason is mainly due to the low solubility of solutes.

The voltammogram of dimethyl sulphoxide contains a reduction peak at around $0.45 \mathrm{~V}$. HEPES and its sodium salt could be dissolved in this liquid in $5 \mathrm{mmol} \cdot \mathrm{L}^{-1}$ concentration. Therefore the reduction peak of HEPES showed up at around $-1.1 \mathrm{~V}$ but did not appear in the voltammogram of its sodium salt indicating that the reduction peak can be attributed to the acidic proton associated with the first dissociation step. An anodic peak appeared at around $0.9 \mathrm{~V}$ in the voltammogram of sodium salt of HEPES attributable to the oxidation of one of the tertiary nitrogen atoms at lower potential due to the identical thermodynamic properties of anionic form compared with HEPES. It could not be observed in acetonitrile due to the poor solubility.

\subsection{Voltammetric Investigation of the Sulphonamide Drugs}

In order to investigate the four selected drugs their voltammograms were recorded between 2 and $-2 \mathrm{~V}$ in their $5 \mathrm{mmol} \cdot \mathrm{L}^{-1}$ solutions prepared with acetonitrile (Fig. 4) 
Fig. 2 Cyclic voltammograms of the solvent acetonitrile and that of acetonitrile solution saturated with solutes (supporting electrolyte $0.05 \mathrm{~mol} \cdot \mathrm{L}^{-1} \mathrm{TBAP}$, scan rate $0.1 \mathrm{~V} \cdot \mathrm{s}^{-1}$ )

Fig. 3 Cyclic voltammograms of the solvent dimethyl sulphoxide and that of dimethyl sulphoxide solution of $5 \mathrm{mmol} \cdot \mathrm{L}^{-1}$ solutes (supporting electrolyte 0.05 $\mathrm{mol} \cdot \mathrm{L}^{-1} \mathrm{TBAP}$, scan rate 0.1 $\left.\mathrm{V} \cdot \mathrm{s}^{-1}\right)$
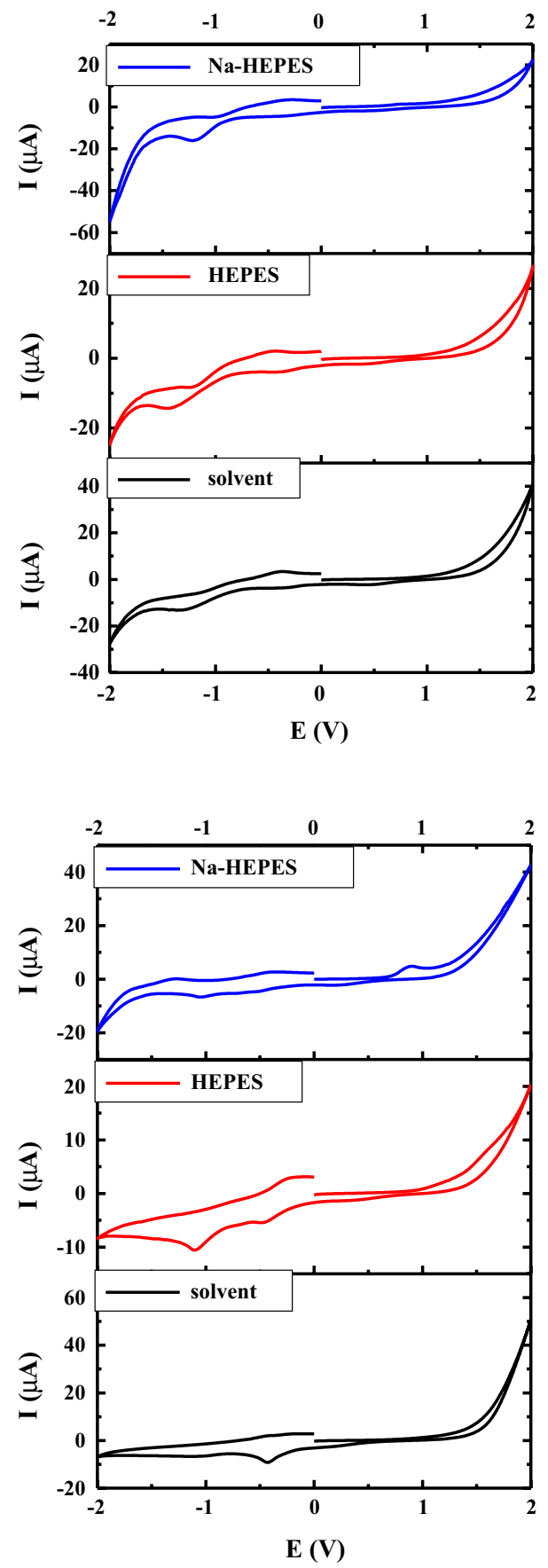

and dimethyl sulphoxide (Fig. 5). The platinum electrode surface had to be polished after each scan to obtain reproducible voltammograms of all sulphonamides as during some subsequent measurement the peak currents declined approximately by $10 \%$. 
Fig. 4 Cyclic voltammograms of the selected sulphonamide drugs in their acetonitrile solution (scan rate $0.1 \mathrm{~V} \cdot \mathrm{s}^{-1}$, supporting electrolyte $0.05 \mathrm{~mol} \cdot \mathrm{L}^{-1} \mathrm{TBAP}$ )
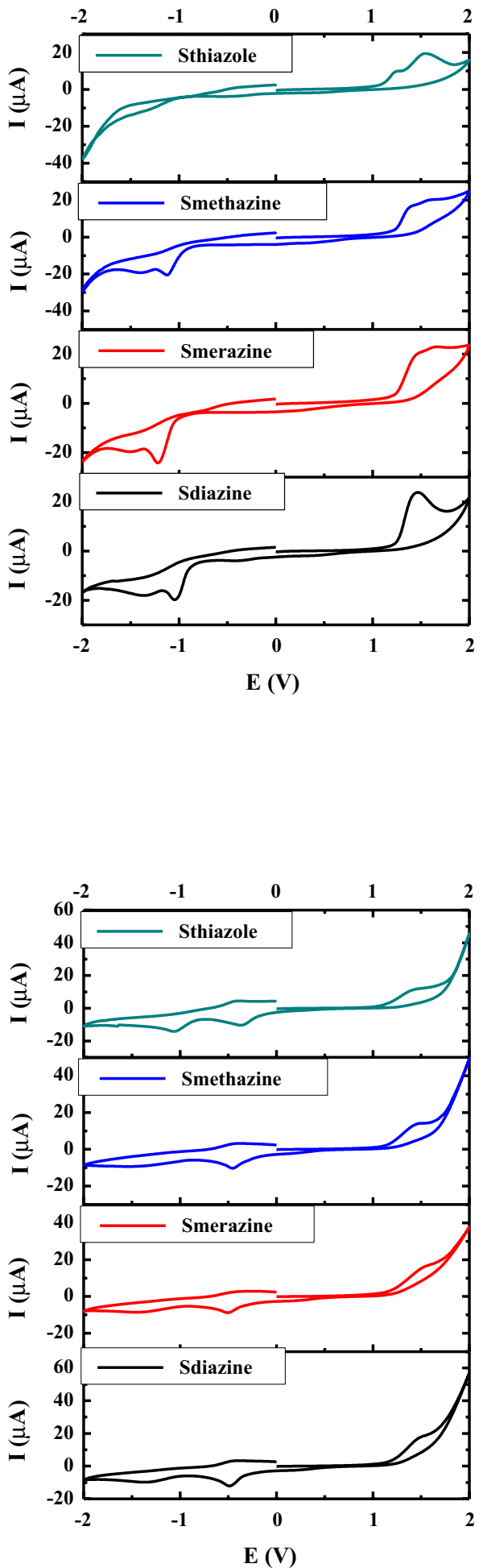

Fig. 5 Cyclic voltammograms of the selected sulphonamide drugs in their dimethyl sulphoxide solution (scan rate $0.1 \mathrm{~V} \cdot \mathrm{s}^{-1}$, supporting electrolyte 0.05 $\left.\mathrm{mol} \cdot \mathrm{L}^{-1} \mathrm{TBAP}\right)$ 
In acetonitrile at around $1.5 \mathrm{~V}$ an anodic peak showed up in case of all drugs where their $\mathrm{NH}_{2}$ - group oxidized. The voltammogram of sulphadiazine contains only one peak around this potential. In this potential region during electrooxidation of sulphamerazine and sulphamethazine an additional peak appeared close to each other the second one possibly assigned to the methyl groups presenting in the pyrazole ring. Sulphathiazole exists in two tautomer forms (imino and amino) and the previous one is dominant (see Fig. 1) [21]. In the imino tautomer an amino group (-NH-) builds up in the thiazole ring being capable of oxidation at lower potentials due to the sulphur atom which increases the electron density on the pyrazole moiety. The tautomerisation is negligible by the other studied sulphonamides. At around $-1.1 \mathrm{~V}$ by appearance of a remarkable cathodic peak electroreduction of acidic proton takes place. The acidic proton of HEPES reduces also here shown previously in dimethyl sulphoxide therefore two overlapping proton reduction process should be considered in the further studies.

The voltammograms recorded in the dimethyl sulphoxide solutions of $5 \mathrm{mmol} \cdot \mathrm{L}^{-1} \mathrm{sul}-$ phonamides were similar to the ones obtained in acetonitrile but the peaks were not sharp. In the anodic part the overlapping with solvent electrooxidation is significant and around $-1.4 \mathrm{~V}$ a small reduction peak showed up. The only exception was sulphatiazole where a well defined peak appeared at around $-1.1 \mathrm{~V}$ due to the reduction of thiazole moiety.

The processes occurring in the system containing the sulphonamides and HEPES as well as its sodium salt was the mostly in focus of interest of the present work. Figure 6 displays the concerning voltammograms for sulphadiazine. By addition of HEPES into the solution containing sulphadiazine similar voltammogram could be obtained as in its absence and only the reduction peak at around $-1.1 \mathrm{~V}$ was a little smaller and peak potential shifted by approximately $20 \mathrm{mV}$, probably due to the protonation of some molecules. The addition of HEPES sodium salt produced the really remarkable change in the voltammogram as the anodic peak of sulphadiazine shifted significantly to a more positive value, more remarkably, the reduction peak at around $-1.1 \mathrm{~V}$ disappeared. These findings suggest that the acid-base reaction between HEPES and sulphadiazine is negligible and another type of interaction develops with Na-HEPES. Basically, in the sodium salt both tertiary amine groups are free, so that they are capable of binding a proton coming from dissociation of the sulphonamide. Therefore the reduction potential of this proton shifts to more negative values. On the other hand few formed HEPES by protonation of its conjugate base stays in solution due to the poor solubility, namely majority of the acidic protons become extracted by the undissolved salt. In HEPES one of the tertiary amine groups is protonated and due to the strong electrostatic repulsion binding of a second proton is prevented. The

Fig. 6 Cyclic voltammograms of $5 \mathrm{mmol} \cdot \mathrm{L}^{-1}$ sulphadiazine in acetonitrile alone (black curve) in presence of HEPES in its saturation concentration (red curve), and in presence of sodium salt of HEPES in its saturation concentration (blue curve) (scan rate 0.1 $\mathrm{V} \cdot \mathrm{s}^{-1}$, supporting electrolyte 0.05 $\left.\mathrm{mol} \cdot \mathrm{L}^{-1} \mathrm{TBAP}\right)$

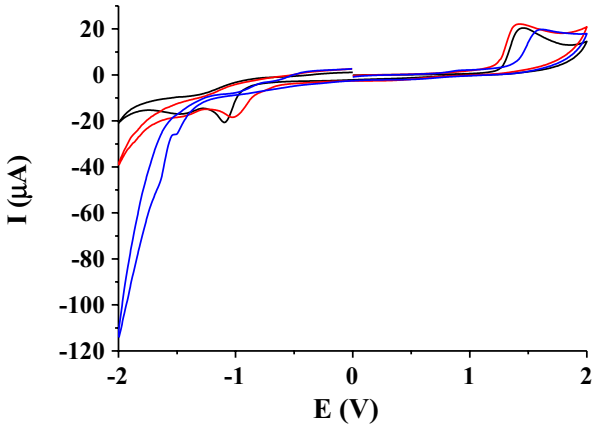


Fig. 7 Cyclic voltammograms of $5 \mathrm{mmol} \cdot \mathrm{L}^{-1}$ sulphadiazine in dimethyl sulphoxide alone (black curve) in presence of HEPES dissolved in $5 \mathrm{mmol} \cdot \mathrm{L}^{-1}$ concentration (red curve), and in presence of sodium salt of HEPES in 5 $\mathrm{mmol} \cdot \mathrm{L}^{-1}$ concentration (blue curve) (scan rate $0.1 \mathrm{~V} \cdot \mathrm{s}^{-1}$, supporting electrolyte $0.05 \mathrm{~mol} \cdot \mathrm{L}^{-1}$ TBAP)

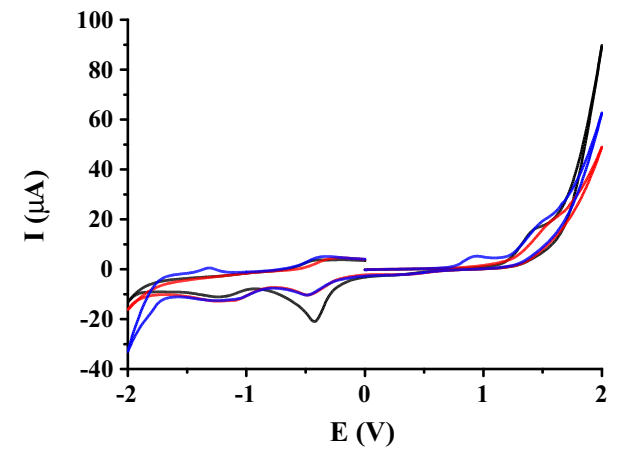

\begin{tabular}{llll}
\hline & Alone & With HEPES & $\begin{array}{l}\text { With Na- } \\
\text { HEPES }\end{array}$ \\
\hline Binary solvent & 1.7 & 3.91 & 406 \\
Sulphadiazine & 4.18 & 5.38 & 373 \\
Sulphamerazine & 3.24 & 4.31 & 378 \\
Sulphamethazine & 2.86 & 4.28 & 381 \\
Sulphathiazole & 2.44 & 4.48 & 370 \\
\hline
\end{tabular}

Table 1 Conductivity results in $\mu \mathrm{S} \cdot \mathrm{cm}^{-1}$ of sulphonamide drugs dissolved in $50-50 \%$ acetonitrile-water mixtures in 5 $\mathrm{mmol} \cdot \mathrm{L}^{-1}$ concentration

previously mentioned positive anodic peak shift serves as an additional verification that majority of sulphonamide molecules become deprotonated. The results were very similar in case of the other three drugs.

The same experiments were repeated in dimethyl sulphoxide for sulphadiazine (Fig. 7). The voltammograms recorded in presence of HEPES and its sodium salt are almost identical around $-1 \mathrm{~V}$ where the reduction peak of HEPES appeared previously when it was present only in solution. This indicates that an acid-base reaction takes place between HEPES and sulphadiazine but not as clearly as in acetonitrile. Basically the other sulphonamides showed similar behavior in presence of HEPES and its sodium salt.

\subsection{Conductometric Studies in Acetonitrile/Water Binary Mixtures}

It was previously mentioned that sulphonamides are scarcely soluble in water. However, water is an interesting solvent of concern in physiological studies, the mixing with acetonitrile as cosolvent was necessary to increase the solubilities of sulphonamides providing a better medium for examination of the processes taking place in the presence of HEPES buffer. On the other hand, water triggers the dissociation of electrolytes due to the increase of the dielectric constant, producing individual ions contributing the increase of conductivity. The presence of water ensures for HEPES and Na-HEPES to be completely dissolved in the solvent mixture as it has low solubility in acetonitrile due to their strong ionic character. The systems where the sulphonamide drugs exist alone and with HEPES as well as its sodium salt were investigated, respectively. The volume ratio of the two solvents was $50-50 \%$. In this binary solvent the drugs were dissolved in equimolar quantity with HEPES or its sodium salt (the solute concentrations were always $5 \mathrm{mmol} \cdot \mathrm{L}^{-1}$ ). The protonation produces ions having the different mobilities than the other ones. Table 1 
shows the conductivity data. In physiological conditions the single deprotonated form of sulphonamides is predominant (the first acidic dissociation step associated with protonated aromatic amino groups) [22] and there is only a small change in conductivities when they are dissolved alone compared with the acetonitrile-water binary solvent. The difference in conductivity between HEPES and Na-HEPES is very significant attributable to the zwitterionic nature of HEPES. By addition of HEPES to the solutions containing the sulphonamides there were no significant differences in the conductivity mainly due to the low degree of ionization of sulphonamide drugs. Na-HEPES does not protonate the drugs but acid-base reactions may occur between them (see previous sections) but its conductivity decreased in the presence of the drugs, indicating that some Na-HEPES becomes protonated producing HEPES zwitterions. More exactly, the acidic protons released by sulphonamides bind to one of the basic nitrogens of HEPES anion which is the basic form of the buffer producing HEPES.

\subsection{Determination of the Solubilities of HEPES and its Sodium Salt in Acetonitrile At $298 \mathrm{~K}$}

As HEPES and its sodium salt are scarcely soluble in acetonitrile their solubilities were determined in it at $298 \mathrm{~K}$. In a previous work the advantages of the co-solvent calibration method were demonstrated in the determination of the solubility of hydroquinone in chlorinated hydrocarbons [23]. This was applied in case of sodium salt of HEPES by using conductometry and on the other hand, as shown in the previous section, its conductivity was high. The uniformity of solvent composition ensures that the baseline for any chosen measurement technique used for concentration determination is the same in each solution. Both solutes are ionic compounds highly soluble in water, therefore high purity water (resistivity approximately $1.5 \mathrm{M} \Omega \cdot \mathrm{cm}$ ) was used as co-solvent, and conductometry was used for the concentration measurements. Water was present in each solution in $50 \mathrm{v} / \mathrm{v} \%$ by solubility determination of sodium salt of HEPES. The calibration solutions were prepared in $25 \mathrm{~cm}^{3}$ flasks by placing $12.5 \mathrm{~cm}^{3}$ of acetonitrile and then the necessary volumes of aqueous stock solution of solute were added. Finally the flasks were filled to the mark with water. The sample solutions were prepared similarly but instead of pure acetonitrile the same volume of saturated solution was placed into the flasks. The measuring part of conductometer was washed thoroughly with pure water. Its traces were removed by washing with pure methanol which was allowed to dry completely. The measured conductivities in the calibration solutions were used to create a calibration curve and finally to determine the solubility. The

Fig. 8 Calibration curve for solubility determination in acetonitrile for Na-HEPES

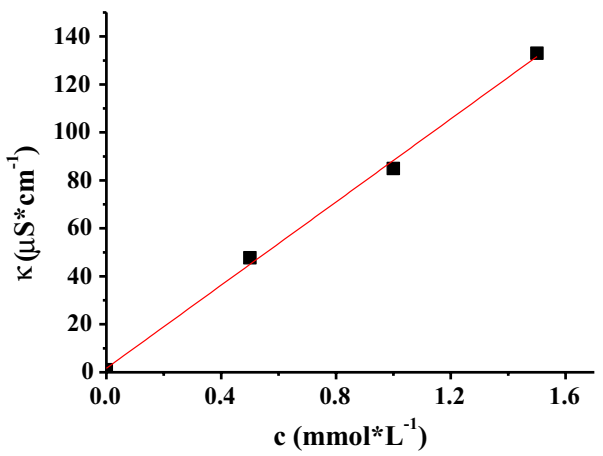


calibration curve is shown in Fig. 8 and it displays linear dependence of specific conductivity on concentration between 0 and $1.6 \mathrm{mmol} \cdot \mathrm{L}^{-1}$ and its calibration equation is $\kappa\left(\mu \mathrm{S} \cdot \mathrm{cm}^{-1}\right.$ )$=1.67+86.64 c\left(\mathrm{mmol} \cdot \mathrm{L}^{-1}\right)\left(\mathrm{R}^{2}=0.9966\right)$. As directly from this equation the concentrations of the diluted solutions can be obtained from the calibration curve it has to be multiplied by 2 to obtain the real solubilities. Three parallel determinations were carried out for $\mathrm{Na}$-HEPES. The obtained values were finally averaged obtaining $0.351 \pm 0.025 \mathrm{mmol} \cdot \mathrm{L}^{-1}$ for its solubility in acetonitrile.

According to the data of Table 1 HEPES has poor conductivity due to its zwitterionic nature so the previously used cosolvent calibration method based on conductometry failed to solve the problem. Therefore, potentiometric $\mathrm{pH}$ titrations were performed. $10 \mathrm{~cm}^{3}$ aliquots of saturated acetonitrile solutions were titrated with $\sim 1 \mathrm{mmol} \cdot \mathrm{L}^{-1}$ aqueous $\mathrm{NaOH}$ titration solution (freshly prepared and determined its exact concentration with $\mathrm{HCl}$ titration solution). The averaging of results of three titrations gave the final quantitative information. At $298 \mathrm{~K}$ the solubility of HEPES in acetonitrile is $0.612 \pm 0.033 \mathrm{mmol} \cdot \mathrm{L}^{-1}$ according to the measurements.

\subsection{Fluorescence Studies}

An additional technique was also used to investigate the interaction between the outlined sulphonamide drugs and HEPES as well as its sodium salt. However, aqueous environment would be really interesting in viewpoint of potential applications each sulphonamides were spectroscopically studied in dimethyl sulphoxide as it has low absorbance in the UV range which was necessary for the excitation. The recording of their UV-VIS spectra provided the wavelength of maximum absorbance $(275 \mathrm{~nm})$. These wavelengths were selected for excitation in the fluorescence studies which was uniform for all sulphonamides except for sulphathiazole whose absorption maximum appeared at $293 \mathrm{~nm}$. The wavelength range used for the fluorescence measurement was between 320 and $400 \mathrm{~nm}$ to minimize the overlap with the spectral range of absorption peaks. Slits at the excitation and detector side of the equipment were set both to $3 \mathrm{~nm}$. The concentration of sulphonamides was $10 \mu \mathrm{mol} \cdot \mathrm{L}^{-1}$ because of the inner filter effect and $100 \mu \mathrm{mol} \cdot \mathrm{L}^{-1}$ for both HEPES and its sodium salt. The corresponding spectra for sulphadiazine are displayed in Fig. 9 which has a visible fluorescence at around $350 \mathrm{~nm}$. In a previous experiment it was established that HEPES

Fig. 9 Fluorescence spectra for $10 \mu \mathrm{mol} \cdot \mathrm{L}^{-1}$ sulphadiazine in dimethyl sulphoxide alone and in presence of HEPES and Na-HEPES. Inset graph shows the absorption spectrum for 1 $\mathrm{mmol} \cdot \mathrm{L}^{-1}$ sulphadiazine in dimethyl sulphoxide

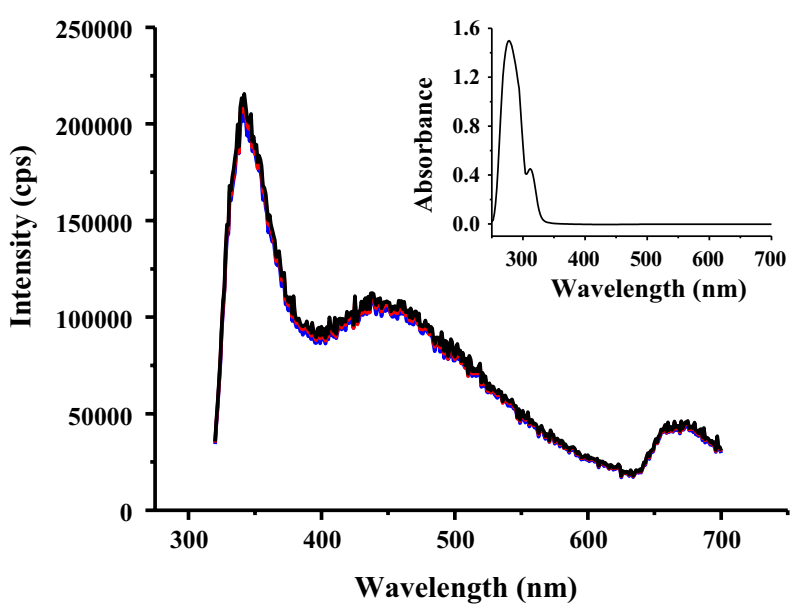


Fig. 10 Normalized fluorescence intensities of sulphonamide drugs in presence of HEPES and its sodium salt

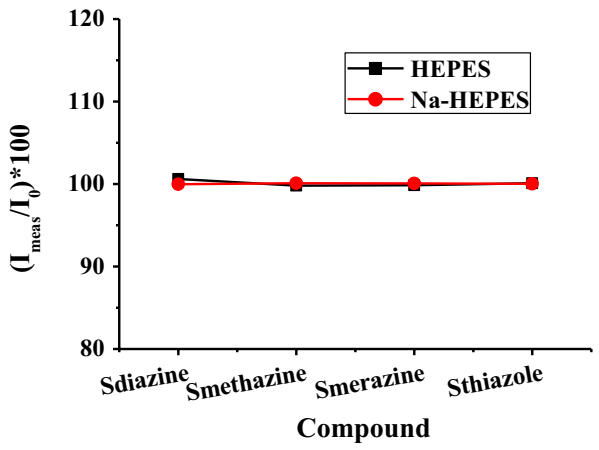

and its sodium salt dissolved in dimethyl sulphoxide in $100 \mu \mathrm{mol} \cdot \mathrm{L}^{-1}$ concentration have negliglible fluorescence intensities in the above spectral ranges. The sulphonamides themselves gave a maximum intensity around 200,000 cps which is not very high compared with other fluorescent materials. The normalized fluorescence intensities $\left(I_{\text {meas }} / I_{\mathrm{o}}\right.$ where $I_{\text {meas }}$ is the intensity in presence of HEPES and its sodium salt, $I_{\mathrm{o}}$ is the intensity in their absence) are plotted in Fig. 10 for the four sulphonamides. Their values are very close to 1 indicating that there is not significant change in the fluorescence intensities suggesting the lack of weak molecular interactions between sulphonamides and HEPES as well as its sodium salt.

\section{Conclusions}

The electrochemical investigations showed that an acid-base reaction takes place between the outlined sulphonamide drugs and sodium salt of HEPES which acts as a basic component. As it could be demonstrated the two non-aqueous solvents together provided results which completed each other providing useful information about studied systems. The used methods highlighted that simultaneous application of sulphonamide drugs and HEPES and NaHEPES causes no complication due to complex formation in biological systems.

Acknowledgements Financial support of the GINOP 2.3.2-15-2016-00022 Grant is highly appreciated.

Funding Open Access funding provided by University of Pécs.

Open Access This article is licensed under a Creative Commons Attribution 4.0 International License, which permits use, sharing, adaptation, distribution and reproduction in any medium or format, as long as you give appropriate credit to the original author(s) and the source, provide a link to the Creative Commons licence, and indicate if changes were made. The images or other third party material in this article are included in the article's Creative Commons licence, unless indicated otherwise in a credit line to the material. If material is not included in the article's Creative Commons licence and your intended use is not permitted by statutory regulation or exceeds the permitted use, you will need to obtain permission directly from the copyright holder. To view a copy of this licence, visit http://creativecommons.org/licenses/by/4.0/. 


\section{References}

1. Rawson, F.J., Cole, M.T., Hicks, J.M., Aylott, J.W., Milne, W.I., Collins, C.M., Jackson, S.K., Silman, N.J., Mendes, P.M.: Electrochemical communication with the inside of cells using micropatterned vertical carbon nanofibre electrodes. Sci. Rep. (2016). https://doi.org/10.1038/srep37672

2. Aeppli, M., Voegelin, A., Gorski, C.A., Hofstetter, T.B., Sander, M.: Mediated electrochemical reduction of iron (oxyhydr-)oxides under defined thermodynamic boundary conditions. Environ. Sci. Technol. 52, 560 (2018)

3. Wang, X., Yang, Y., Li, L., Sun, M., Yin, H., Qin, W.: A polymeric liquid membrane electrode responsive to 3,3',5,5'-tetramethylbenzidine oxidation for sensitive peroxidase/peroxidase mimetic-based potentiometric biosensing. Anal. Chem. 86, 4416 (2014)

4. Pimentel, D.M., Silva, S.M., Leite, F.R.F., Tanaka, A.A., Santos, W.T.P., Damos, F.S., Luz, R.C.S.: Derivative potentiometric stripping analysis of $\mathrm{Cu}$ (II) at nanomolar levels exploiting the 4-[10,15,20-tris(4-sulfophenyl)-21,22-dihydroporphyrin-5-yl] benzenesulfonic acid host compound on multi-walled carbon nanotubes. Int. J. Electrochem. Sci. 8, 6131 (2013)

5. Santos, T.A.D., Neto, S.Y., Macena, C.S., Luz, R.C.S., Damos, F.S.: Photoelectroanalytical detection of adrenaline based on DNA and $\mathrm{TiO}_{2}$ nanoparticles sensitized with bis(ethylenedithio)tetrathiafulvalene exploiting LED light. J. Braz. Chem. Soc. 28, 1752 (2017)

6. Joel, S., Turner, K.B., Daunert, S.: Glucose recognition proteins for glucose sensing at physiological concentrations and temperatures. ACS Chem. Biol. 9, 1595 (2014)

7. Momberg, V.A., María, E.C.B., von Baer, D., Bruhn, F.C., Smyth, M.R.: The oxidative voltammetric behaviour of some sulphonamides at the glassy carbon electrode. Anal. Chim. Acta 159, 119 (1984)

8. Nikoofard, H., Sargolzaei, M., Faridbod, F.: Prediction of physico-chemical properties of bacteriostatic N1-substituted sulfonamides: theoretical and experimental studies. Acta Chim. Slov. 64, 842 (2017)

9. Andrade, L.S., Rocha-Filho, R.C., Cass, Q.B., Fatibello-Filho, O.: Simultaneous differential pulse voltammetric determination of sulfamethoxazole and trimethoprim on a boron-doped diamond electrode. J. Electroanal. Chem. 21, 1475 (2009)

10. Lahcen, A.A., Errayess, S.A., Amine, A.: Voltammetric determination of sulfonamides using paste electrodes based on various carbon nanomaterials. Microchim. Acta 183, 2169 (2016)

11. Carrazon, J.M.P., Recio, A.D., Diez, L.M.P.: Electroanalytical study of sulphamerazine at a glassycarbon electrode and its determination in pharmaceutical preparations by HPLC with amperometric detection. Talanta 39, 631 (1992)

12. Carrazon, J.M.P., Corona, P.C., Diez, L.M.P.: Electroanalytical study of sulphadiazine at solid electrodes. Determination in pharmaceutical preparations. Electrochim. Acta 32, 1573 (1987)

13. Rao, T.N., Sarada, B.V., Tryk, D.A., Fujishima, A.: Electroanalytical study of sulfa drugs at diamond electrodes and their determination by HPLC with amperometric detection. J. Electroanal. Chem. 491, 175 (2000)

14. Braga, O.C., Campestrini, I., Vieira, I.C., Spinelli, A.: Sulfadiazine determination in pharmaceuticals by electrochemical reduction on a glassy carbon electrode. J. Braz. Chem. Soc. 21, 813 (2010)

15. Eleotério, I.C., Balbino, M.A., Andrade, J.F., Ferreira, B., Saczk, A.A., Okumura, L.L., Batista, A.C.F., Oliveira, M.F.: Analysis of sulfamethoxazole by square wave voltammetry using new carbon paste electrode. J. Electrochem. Sci. Eng. 8, 281 (2018)

16. Ghoreishi, S.M., Behpour, M., Khoobi, A., Masoum, S.: Application of experimental design for quantification and voltammetric studies of sulfapyridine based on a nanostructure electrochemical sensor. Arab. J. Chem. 10, S3156 (2014)

17. Dogan-Topal, B., Ozkan, S.A., Uslu, B.: The analytical applications of square wave voltammetry on pharmaceutical analysis. Open Chem. Biomed. Methods J. 3, 56 (2010)

18. Fogg, A.G., Rahim, H.A., Yusoff, M., Moreira, J.C., Chao, R.: Cathodic stripping voltammetric determination of sulfonamides as copper(I) complexes at a hanging mercury drop electrode. Anal. Proc. 32, 95 (1995)

19. Mabrouk, E.M., Al-Omary, Kh.A., Al-Omary, A.S., El-Mossalamy, E.H.: Electrochemical and spectral studies of some sulfa drug azodyes and their metal complexes in aqueous solution. J. Adv. Chem. 14, 6150 (2017)

20. Zigler, J.S., Lepe-Zuniga, J.L., Vistica, B., Gary, I.: Analysis of the cytotoxic effects of light-exposed hepes-containing culture medium. In Vitro Cell. Dev. Biol. 21, 282 (1985)

21. Kruger, G.J., Gafner, G.: The crystal structure of sulphathiazole II. Acta Crystallogr. B27, 326 (1971)

22. Qiang, Z., Adams, C.: Potentiometric determination of acid dissociation constants $\left(\mathrm{p} K_{\mathrm{a}}\right.$ ) for human and veterinary antibiotics. Water Res. 38, 2874 (2004) 
23. Kiss, L., Kunsági-Máté, S.: Solubility determination of hydroquinone in dichloromethane, trichloromethane and carbon tetrachloride by using the co-solvent calibration method. J. Solution Chem. 48, 1357 (2019)

Publisher's note Springer Nature remains neutral with regard to jurisdictional claims in published maps and institutional affiliations. 